\title{
Energy transfer in a liquid filled elemental passage of a porous medium for permeability enhancement due to pulsations of a vapor bubble
}

\author{
F. Rambarzin*, M. T. Shervani-Tabar**, M. Taeibi-Rahni***, S. A. Tabatabaei-Nejad**** \\ *Department of Mechanical and Aerospace Engineering, Science and Research branch, Islamic Azad University, Tehran, \\ Iran, E-mail: f.rambarzin@srbiau.ac.ir \\ **Department of Mechanical Engineering, University of Tabriz, Tabriz, Iran, E-mail: msherv@tabrizu.ac.ir \\ ***Department of Aerospace Engineering, Sharif University of Technology, Tehran, Iran, E-mail: taeibi@sharif.edu \\ ****Department of Petroleum Engineering, Sahand University of Technology, Tabriz, Iran, E-mail: tabatabaei@ sut.ac.ir \\ cross $^{\text {ref }}$ http://dx.doi.org/10.5755/j01.mech.22.1.14238
}

\section{Introduction}

Enhancing Oil Recovery (EOR) is an important goal in the petroleum industries and has important effect on the development of the nations from the Economical and industrial points of view. In this paper the boundary integral equation method has been employed for numerical simulation of the growth and collapse of a vapor bubble in a narrow cylindrical passage due to the emission of the ultrasound waves.

The growth and collapse phases of the ultrasound induced cavitation bubbles in the liquid domain filled in the passages of a porous medium, transfer the energy of the emitted ultrasound waves in the liquid domain in an explosive manner and consequently apply impact forces on any obstacles which may exist or be produced during the operating time of the oil well in the passages of the porous medium and consequently reduce its permeability. The impact forces on these obstacles which are the consequent of the energy transfer through the velocity and pressure fields in the liquid filled passages of the porous medium due to the explosive pulsations of the vapor bubbles could remove these obstacles and increase the permeability of the porous medium.

Conventional methods for oil enhancing recovery like hydraulic fracture, $\mathrm{CO}_{2}$ injection, steam flooding and hot water flooding, have been known for a long time in the petroleum industries [1-5].

During the last decade employing of ultrasound waves for increasing permeability of a porous medium and consequently enhancing oil recovery has been subject of a few researches throughout the world [6-11]. By applying ultrasound waves to a porous medium filled with different liquids, cavitation bubbles are generated in the liquids inside the passages of the porous medium.

Because of the important roles of the cavitation bubble in enhancing oil recovery and cracking of heavy crude oil and other processes in petroleum and petrochemical industries, medicine, and other fields of science and industry, the interested researchers in this field have attempted to understand the dynamic behavior of vapor cavitation bubble in the narrow confined regions via experimental and numerical investigations [12-14].

These highly pulsating bubbles in the liquid filled passages of the porous medium transfer the energy of the ultrasound waves through the liquid domain of their periphery via highly dynamic velocity and pressure fields and have high impact forces on the obstacles existing in the passages of the porous medium. These obstacles have been accumulated in the passages of the porous medium during the operation time of the oil wells and act as plugs in these passages and consequently reduces permeability of the porous medium. Therefore highly dynamic impact forces acting due to the explosive pulsations of the ultrasound waves induced by cavitation bubbles could remove these obstacles and consequently lead to the increasing of permeability of the porous medium and enhancing oil recovery. An experimental study in the Penn state university in 2004 [15], and few experimental and numerical researches in Canada [16-17], Russia [18] and Malaysia [19] have been reported about application of ultrasound waves for enhancing oil recovery.

In this paper a fundamental numerical study has been reported for understanding of some of the mechanisms of oil recovery enhancement by using the ultrasound waves. Three different axisymmetric passages are introduced as geometrical models for the passages of a porous medium. A boundary integral equation method based on the Green's integral formula and potential fluid flow theory has been employed for the numerical simulation of this problem.

\section{An important note on the strategy of the solution of this research}

By the emission of the ultrasound waves to a liquid filled porous medium, energy of the ultrasound waves transfer to the porous medium. Some fraction of this energy dissipate in the solid portion of the porous medium and in the bubbly liquid flow domain inside the passages of the porous medium and some other fraction of the energy of the ultrasound waves have been used for generation of the cavitation vapor bubbles in the liquid filled passages throughout the porous medium. The generation of the cavitation bubbles starts from the nuclei hosts of the liquid domain. The initial boundary conditions for growing of a cavitation bubble are based on the distribution of the velocity potential on the initial boundary of the minimum vapor bubble. The magnitude of the velocity potential on the boundary of initial vapor bubble can be obtained by using Beta incomplete function.

Blake et al. [20] clearly explained the initial boundary conditions of the cavitation bubbles. Best in his novel work gives the theory for the initial boundary conditions of the explosion bubbles [21].

In this research, the energy of the ultrasound 
waves has been considered for generation of the cavitation bubbles and consequently enhancing oil recovery. It should be noted that the mechanisms of the growth and collapse of the cavitation and explosion bubbles are mostly the same. During the growth and collapse phases of a cavitation bubble, the vapor pressure inside the bubble is constant and is equal to the saturated vapor pressure. The velocity potential on the boundary of a cavitation bubble in its minimum volume can be calculated by using Beta incomplete function. But the pressure inside an explosion bubble during its growth and collapse phases is variable and could be calculated from the thermodynamic isentropic equation for an ideal gas approximately. In this paper, the theory of the explosion bubbles which is given by Best [21] is employed for the growth and collapse phases of the bubbles induced by ultrasound waves in the liquid filled passages of a porous medium. This choice enables us to evaluate clearly the energy input for generation of each bubble. Employing of Beta incomplete function and the initial boundary condition for the cavitation bubbles which are given by Blake et al. [20] remains as our future duty.

\section{Geometry of the problem: an elemental model of a porous medium}

The geometry of problem is shown in Fig. 1.

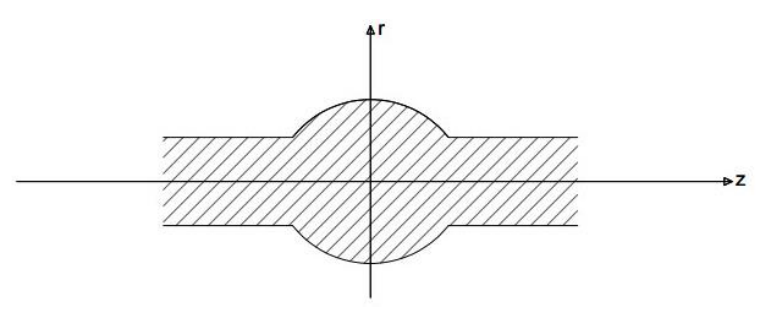

Fig. 1 Schematic representation of a small portion of a porous medium in a petroleum reservoir

Fig. 1 illustrates a small portion of a porous medium of a petroleum reservoir. As it is shown in Fig. 1, the problem is axisymmetric and symmetric axis assumed to be horizontal. The horizontal axisymmetric passage represented in this figure is filled with an inviscid and incompressible liquid which could be brine.

Fig. 2 shows a bubble in its initial minimum volume which is generated due to a local energy input and is located at the center of the liquid filled area of the passage, assumed as a small portion of the porous medium.

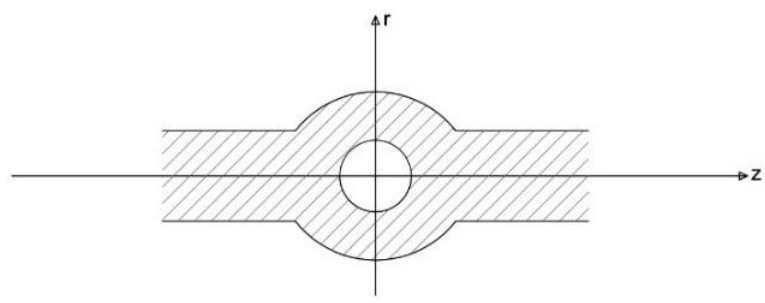

Fig. 2 Schematic representation of a vapor bubble generated due to a local energy input and located at the center of the liquid filled passage of a porous medium

\section{Governing equations}

The liquid in the horizontal passage of Fig. 1 is brine and is assumed to be inviscid and incompressible. A small spherical vapor bubble with an internal high pressure and high temperature is initially generated at the center of the liquid filled horizontal passage due to a local energy input. The liquid flow around the vapor bubble is considered as an irrotational flow and then the flow around the vapor bubble is a potential flow.

The equation which explains the motion of a spherical vapor bubble in its initial state with a given initial pressure is given as:

$$
\left.\begin{array}{l}
R \ddot{R}+\frac{3}{2} \dot{R}^{2}+\frac{\Delta P}{\rho}=0 ; \\
\Delta P=P_{\infty}-P_{c},
\end{array}\right\}
$$

where $\Delta P$ is difference of the pressure in the far field $\left(P_{\infty}\right)$ and saturated vapor pressure $\left(P_{c}\right), R$ is the radius of the bubble and dots denote derivative with respect to time, thus $\dot{R}$ is the radial velocity of the bubble andis the $\ddot{R}$ radial acceleration [22].

The Green's integral formula governing the potential liquid flow around the vapor bubble is given by:

$$
\begin{aligned}
& C_{p} \Phi(p)+\int_{s} \Phi(q) \frac{\partial}{\partial n}\left(\frac{1}{|p-q|}\right) d s= \\
& =\int_{s} \frac{\partial}{\partial n} \Phi(q) \frac{1}{|p-q|} d s,
\end{aligned}
$$

where $p$ is a point in the liquid domain or on its boundary and $q$ is a point on the boundaries of the liquid domain, $\Phi$ is the velocity potential, $C_{p}$ is a constant parameter and $\frac{\partial \Phi}{\partial n}$ is the normal velocity on the boundaries of the liquid domain. The unsteady Bernoulli's equation is used for time historical evaluation of the velocity potential and is given as:

$$
P_{b}=P_{\infty}-\rho \frac{\partial \Phi}{\partial t}-\frac{1}{2} \rho|\bar{u}|^{2}
$$

\section{Discretization of the problem}

Fig. 3 illustrates the discretized boundaries of the selected elemental model of a porous medium.

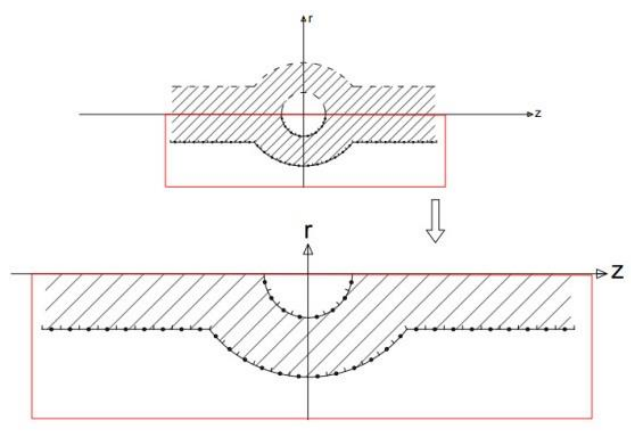

Fig. 3 Schematic representation of discretized boundaries of the selected elemental model of a porous medium

Since the problem is axisymmetric then the symmetric axis behaves as a mathematical boundary and the boundaries at the two extreme ends of the liquid domain 
are assumed to be at physical infinity. Thus the domain of this study is a bounded domain, which is required for satisfying of the conservation laws.

The Green's integral formula which is mentioned in Eq. (2) discretized as follow:

$$
\left.\begin{array}{l}
C_{p_{i}} \Phi\left(p_{i}\right)+\sum_{j=1}^{m} \int_{s_{j}} \Phi\left(q_{j}\right) \frac{\partial}{\partial n} \frac{1}{\left|p_{i}-q_{j}\right|} d s= \\
=\sum_{j=1}^{m} \int_{s_{j}} \frac{\partial}{\partial n} \Phi\left(q_{j}\right) \frac{1}{\left|p_{i}-q_{j}\right|} d s ; \\
C_{P_{i}}=2 \pi \text { if } p_{i} \in S ; \\
C_{P_{i}}=4 \pi \text { if } p_{i} \in \Omega,
\end{array}\right\}
$$

where $S$ is the surface of the liquid domain and $\Omega$ is liquid domain. The unsteady Bernoulli's equation for any points on the moving boundaries of the liquid domain in the Lagrangian form is given:

$$
\frac{D \Phi}{D t}=\frac{P_{\infty}-P_{b}}{\rho}+\frac{1}{2}|\nabla \Phi|^{2},
$$

where $P_{b}$ is the variable pressure inside the vapor bubble, generated due to a high local energy input, during its growth and collapse phases.

The discretized form of unsteady Bernoulli's equation in its Lagrangian form is given as:

$$
\frac{\Phi_{j}^{n+1}-\Phi_{j}^{n}}{\Delta t}=\frac{P_{\infty}-P_{b}}{\rho}+\frac{1}{2}\left|\nabla \Phi_{j}^{n}\right|^{2}
$$

The problem is non-dimensionalised based on the following parameters, the maximum radius of the bubble $R_{m}$, the difference of the pressure in the far field $\left(P_{\infty}\right)$ and saturated vapor pressure, $\Delta P$, the density of the fluid, $\rho$.

The non-dimensional parameters are: $R_{m}\left(\frac{\rho}{\Delta P}\right)^{\frac{1}{2}}$ for time, $R_{m}\left(\frac{\Delta P}{\rho}\right)^{\frac{1}{2}}$ for the velocity potential and $\left(\frac{\Delta P}{\rho}\right)^{\frac{1}{2}}$ for the normal velocity on the boundary of the bubble.

Here, by assuming the non-condensable gas inside the vapor bubble as an ideal gas which goes an isentropic process and by employing the above defined nondimensional parameters, Eq. (1) becomes:

$$
\begin{aligned}
& \bar{R} \overline{\ddot{R}}+\frac{3}{2} \overline{\dot{R}}^{2}=\varepsilon\left(\overline{\frac{R_{0}}{\bar{R}}}\right)^{3 \gamma}-1 ; \\
& \varepsilon=\frac{P_{0}}{\Delta P},
\end{aligned}
$$

where $\varepsilon$ is called the strength parameter, which is a measure of the strength of the initial high pressure inside the vapor bubble and enables the bubble to grow to its maximum volume, $\gamma$ is the Ratio of the specific thermal coefficient of the gas inside the bubble, $P_{0}$ is the Initial high pressure inside the bubble.

\section{Theoretical scenario for solution of the problem and its computational implementation}

For the solution of the problem under investigation a theoretical scenario has been developed based on the idea that the input energy generates a very small vapor bubble with a very high pressure and temperature in its inside. At the initial instant the vapor bubble is spherical in its minimum volume and the radial velocity of its surface is assumed to be zero.

Although the radial velocity of the initial spherical bubble in its minimum volume is zero, but due to the high pressure and temperature of its internal vapor contents, its spherical surface has a high acceleration and starts to grow. These has three different geometrical shapes of horizontal passages are constructed as elemental models of small portions of a porous medium which provide different confined spaces around a vapor bubbles generated by a local energy input.

The computational implementation of simulation of the growth and collapse phases of the vapor bubbles inside each of the three different horizontal passages which have been proposed as elemental models for a porous medium in this research is as explained below.

Distribution of the velocity potential on the minimum spherical vapor bubble initially is known an all velocity potential located at the middle point of each linear elements are equal to zero. Also the normal velocity on each linear element on the wall of the horizontal passage is zero. Then by solving the Green's integral formula the normal velocities on the bubble surface and velocity potentials on the linear elements along the wall of the passage can be obtained.

By defining a variable time step as below the evolution of the vapor bubble inside, the passages which have been proposed as elemental models representing a small portion of a porous medium can be explained below:

$$
\Delta t=\min \left|\frac{\Delta \Phi}{\frac{P_{\infty}-P_{b}}{\rho}+\frac{1}{2}\left|\nabla \Phi_{j}^{n}\right|^{2}}\right|,
$$

where $\Delta \Phi$ is some constant that represents the maximum increase of the velocity potential on the bubble boundary between the two successive time steps.

\section{Numerical results and discussion}

Fig. 4 illustrates time dependent profiles of the vapor bubble during its growth and collapse phases in a cylindrical passage. As it is seen in this figure, the bubble during its growth phase elongates along the axis of symmetry and then the liquid particles in the vicinity of the vapor bubble have been accelerated in the direction away from the bubble in its both left and right sides. At the end of the growth phase the dynamic pressure of the accelerated liquid particles has been converted to the static pressure. Therefore two high pressure regions in the left and right sides of the elongated bubble in its maximum volume are developed. These high pressure regions in the left and right sides of the bubble cause development of the liquid jets on the right and left extreme boundaries of the bubble with a 
very high velocity and convert the vapor bubble to a toroidal bubble. Then two high pressure regions in the both left and right sides of the bubble coincide each other in the centroid of the toroidal bubble and cause development of a disk shape liquid jet which is directed to the cylindrical wall. The development of the disk shape liquid jet has been reported by Farhangmehr et al. [2014]. Converting the vapor bubble into a toroidal bubble is beyond the scope of this work.

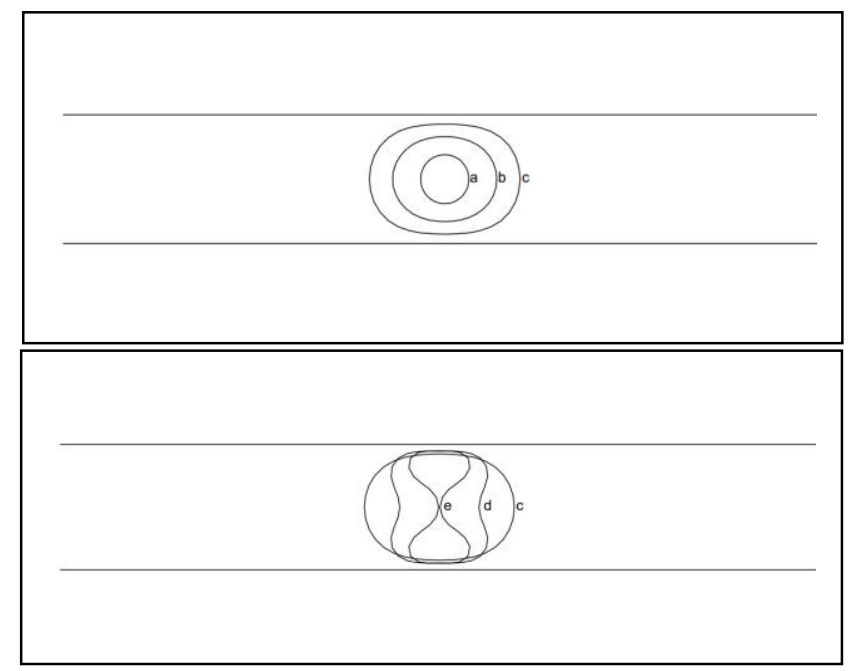

Fig. 4Time-dependent profiles of a vapor bubble generated due to a local energy input in the cylindrical capillary tube in a porous medium. Corresponding nondimensional times are: a) $t=0.00364$; b) $t=0.70303 ; \quad$ c) $t=2.14653 ; \quad$ d) $t=3.84178$; e) $t=4.79135$

Fig. 5 illustrates the time dependent profiles of the vapor bubble during its growth and collapse phases in a cylindrical passage which has a semi-tube like cavity around the vapor bubble. Then in the case of Fig. 5, the vapor bubble during its growth and collapse is not in a space confined as much as in the case of Fig. 4. Due to the this fact in Fig. 5, it is shown that the vapor bubble at the end of its growth phase is not as much elongated as in the case of Fig. 4. Therefore the liquid particles around the axisymmetric axis in the direction away from the bubble are not as high accelerated as in the case of Fig. 4. Consequently the magnitude of the pressure at the left and right sides of the bubble are not as high as the case of Fig. 4. Thus with this background of physics of the liquid flow two liquid jets which are developed at both left and right sides of the bubble are broader in comparison with the case of figure 4 .

Fig. 6 illustrates the growth and collapse phases of a vapor bubble in a cylinder passage with an elliptical semi-tube like axisymmetric cavity. It seems that the conditions of Fig. 6 from the confined space point of view around the vapor bubble is between the cases of Figs. 4 and 5. Therefore two developed liquid jets on both extreme left and right sides of the vapor bubble boundary during its collapse phase are more broader than the developed liquid jets of the case of figure 4 and less broader than the developed liquid jets of the case of Fig. 5. Thus in the case of Fig. 6, the exerted forces on the obstacles which may exist in front of the bubble induced liquid flow due to the dynamic velocity and pressure fields of the liquid domain are higher than the exerted forces on the obstacles which may exist in front of the bubble induced liquid flow in the case of Fig. 4 and is lower than the corresponding forces in the case of Fig. 5.

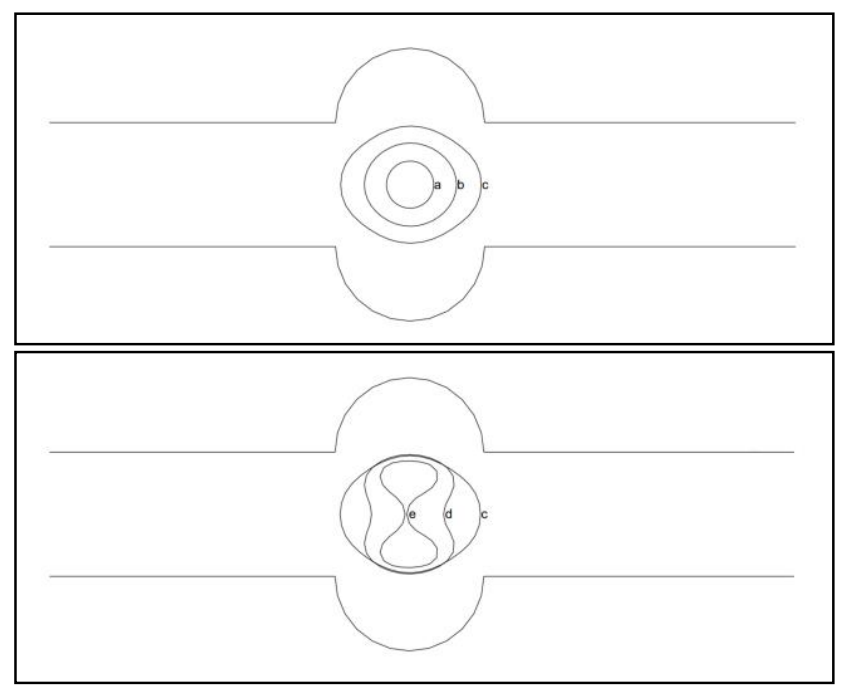

Fig. 5 Time-dependent profiles of a vapor bubble generated due to a local energy input in the hemispherical pore in the middle of the passage in a porous media. Corresponding non-dimensional times are: $\begin{array}{lll}\text { a) } t=0.00364 ; & \text { b) } t=0.60248 ; & \text { c) } t=2.04458 \text {; }\end{array}$ d) $t=3.76093$; e) $t=4.62447$

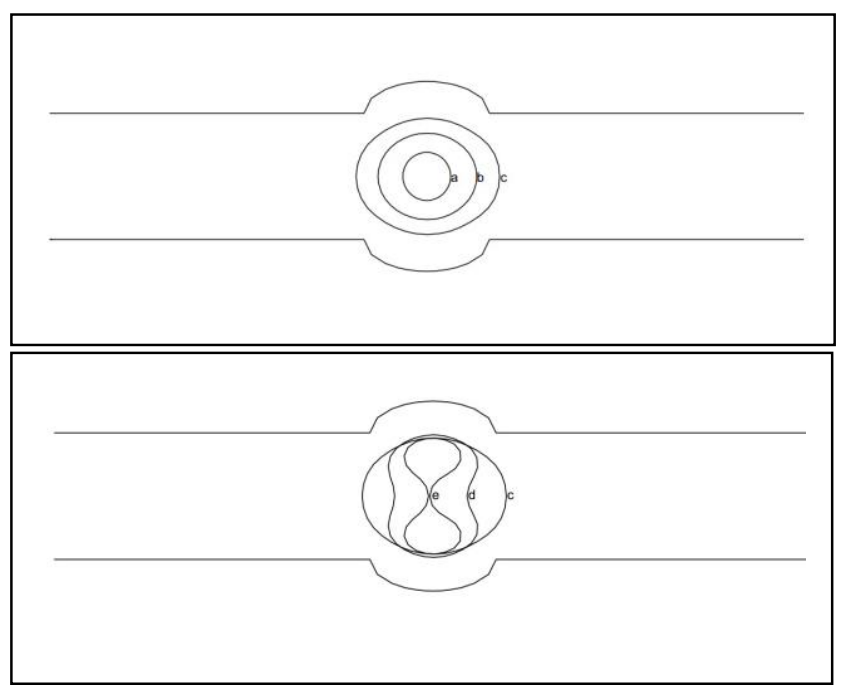

Fig. 6 Time-dependent profiles of a vapor bubble generated due to a local energy input in the semi-ellipsoidal pore in the middle of the passage in a porous media. Corresponding non-dimensional times are:
a) $t=0.00364$;
b) $t=0.68407$;
c) $t=2.16404$;
d) $t=3.87322$; e) $t=4.67378$

Fig. 7 illustrates the velocity of the liquid jets which are developed during the collapse phases of the vapor bubble in the above three different geometrical elements of a porous medium. As it is seen in this figure, in the case of a vapor bubble in a cylindrical passage which provides the most confined space around the vapor bubble during its growth and collapse phases, the bubble during its growth phase elongates along the symmetry axis of the cylinder more than the other two cases. Thus, in this case the liquid particles in the right and left sides of the bubble 
along the symmetry axis of the passages accelerates more than the two other cases. Consequently high pressure regions developed in the right and left sides of the vapor bubble at the end of its growth phase, because of bringing to rest of the accelerated liquid particles have the highest magnitude of the pressure in comparison with the two other cases. Thus having the highest magnitude of the pressure regions in the right and left sides of the bubble at the end of its growth phase inside a cylindrical passage, results in having the highest magnitude of the liquid jet velocities at the instant of transforming the vapor bubble to a toroidal bubble.

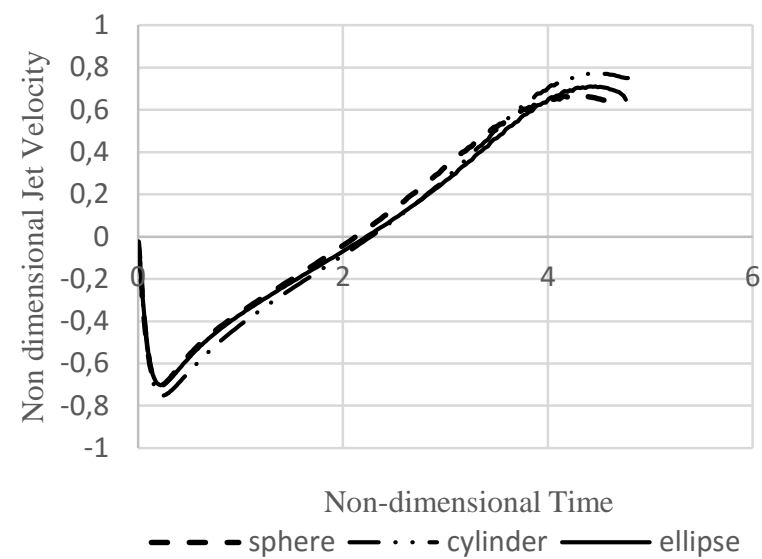

Fig. 7 Variation of the non-dimensional jet velocity at the right and left sides of the vapor bubble against nondimensional time during its growth and collapse phases for the cases of 1 cylinderical passage, 2 cylinderical passage with the hemispherical pore in the middle of the passage, 3 cylinderical passage with the semi-ellipsoidal pore in the middle of the passage

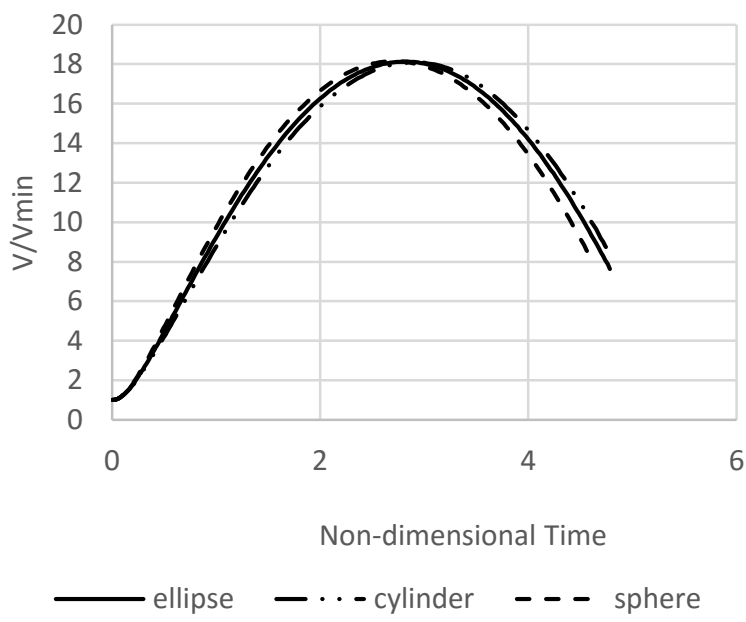

Fig. 8 Variation of the ratio of the bubble volume to its minimum volume against the non-dimensional time during its growth and collapse phases for the cases of 1 cylinderical passage, 2 cylinderical passage with the semi-spherical pore in the middle of the passage, 3 cylinderical passage with the semiellipsoidal pore in the middle of the passage

Fig. 8 illustrates rate of the growth and collapse phases of the bubble in a: 1 - cylindrical passage, 2- cylin- drical passage with a semi-spherical pore in the middle of the passage, 3-cylindrical passage with a semi-ellipsoidal pore in the middle of the passage. As it has been shown in Fig. 8 the growth and collapse rate of the vapor bubble in the case of a cylindrical passage is the lowest and the rate of the growth and collapse phases of the vapor bubble in the case of a cylindrical passage with a semi-spherical pore in the middle of the passage is the highest. Also Fig. 8 shows that the rate of the growth and collapse phases of the vapor bubble in the case of a cylindrical passage with a semi-ellipsoidal pore in the middle of the passage is between the two extreme cases. Fig. 8 illustrates the fact that the most confined space increases the lifetime of a vapor bubble during its growth and collapse phases.

\section{Concluding remarks}

In this paper the idea of Enhancing of Oil Recovery (EOR) is investigated numerically in a novel approach by proposing three different geometries as elemental geometrical models for a porous medium. This novel approach is based on the idea of transferring of energy of the ultrasound waves to liquid filled passages of a porous medium by explosive growth of cavitation vapor bubbles and their consequent violate collapse. The highly explosive pulsations of the vapor bubbles, produce highly dynamic velocity and pressure fields in the liquid domain inside the passages of the porous medium and apply high impact forces on the obstacles which may exist in the passages in front of the bubble induced liquid flow and could remove them. Thus removing of these obstacles in the passages of the porous medium increases the permeability of the porous medium of the oil reservoir and consequently results in Enhancing of Oil Recovery.

\section{References}

1. Green, D.W.; Wilhite, G.P. 1998. Enhanced Oil Recovery, Textbook Series, SPE, Richardson, 545 p.

2. Seryght, R.S. 1983. The effect of mechanical degradation and viscoelastic behavior on injectivity of polyacrylamide solutions, Journal of SPE 23(3): 475-485. http://dx.doi.org/10.2118/9297-PA.

3. Verma, M.K. 2015. Fundamentals of carbon dioxideenhanced oil recovery (CO2-EOR): U.S. Geological Survey Open-File Report 2015-1071, 19 p. http://dx.doi.org/10.3133/ofr20151071.

4. Gaddle, C.; Castenier, M.L. 1991. Basic concepts in enhanced oil recovery process-hot fluid injection, Edited By Baviere, M., Published for SCI by Applied Science, London and New York.

5. Parts, M. 1982. Thermal Recovery, SPE Monograph Series Vol. 7, 283 p.

6. Zaslavskii, Y.M. 2002. Experimental study of the motion of liquid droplets in a capillary under vibration, Acoustical Physics 48(1): 50-53. http://dx.doi.org/10.1134/1.1435389.

7. Li, W.Q.; Vigil, R.D.; Beresnev, L.A.; Lassonov, P.; Ewing, R. 2005 Vibration-induced mobilization of trapped oil ganglia in porous media: Experimental validation of a capillary-physics mechanism, Journal of Colloid and Interface Science 289(1): 193-199. http://dx.doi.org/10.1016/j.jcis.2005.03.067.

8. Poesio, P.;Ooms, G. 2004. Formation and ultrasonic 
removal of fouling particle structures in a natural porous material, Journal of Petroleum Science and Engineering 45(3-4): 159-178.

http://dx.doi.org/10.1016/j.petrol.2004.07.008.

9. Wong, S.W.; Van der Bas, F.; Zuiderwijk, P. 2004. High-power/high-frequency acoustic stimulation: A novel and effective wellbore stimulation technology, SPE Production \& Facilities 19(4): 183-188. http://dx.doi.org/10.2118/84118-MS.

10. Bjorndalen, N.; Islam, M. R. 2004. The effect of microwave and ultrasonic irradiation on crude oil during production with a horizontal well, Journal of Petroleum Science and Engineering 43(3-4): 139-150. http://dx.doi.org/10.1016/j.petrol.2004.01.006.

11. Tu, X.; Ooms, G.; Van Der Tu, F. 2007. Experimental evidence of ultrasonic stimulation of brine-oil flow through a porous rock in laboratory conditions, Transp. Porous Med. 70: 323-333. http://dx.doi.org/10.1007/s11242-007-9102-y.

12. Shervani-Tabar, M.T.; Rambarzin, F.; Shabgard, M.R.; Payamani, R. 2011. Numerical study on the dynamics of an electrical discharge generated vapor bubble in EDM with different shapes of the tool and the workpiece, Int. J. Adv. Manuf. Technol. 56(1-4): 151159. http://dx.doi.org/10.1007/s00170-011-3154-4.

13. Zhang, A.M.; Ni, B.Y.; Song, B.Y.; Yao, X.L. 2010. Numerical simulation of the bubble breakup phenomena in the narrow flow field, Applied Mathematics and Mechanics 31: 420-432. http://dx.doi.org/10.1007/s10483-010-0405-z.

14. Ni, B.Y.; Zhang, A.M.; Wang, Q.X.; Wang, B. 2012. Experimental and numerical study on the growth and collapse of a bubble in a narrow tube, Acta Mechanica Sinica 28(5): 1248-1260. http://dx.doi.org/10.1007/s10409-012-0147-y.

15. Johnson, D.O.; Edgar, D.E.; Wilkey, M.L.; Paulsen, P.D.; Greer, A.W. 2004. Sonication stimulation of stripper well production in East Gilbertown field, WestCentral Alabama, Final Report, The Pennsylvania State University, Subcontract No.2548-TSI-DOE 1025.

16. Hamida, T.; Babadagli, T. 2007. Analysis of capillary interaction and oil recovery under ultrasonic waves, Transp. Porous Med. 70: 231-255. http://dx.doi.org/10.1007/s11242-006-9097-9.

17. Naderi, Kh.; Babadagli, T. 2011. Pore-scale investigation of immiscible displacement process in porous media under high-frequency sound waves, Journal of Fluid Mechanics 680: 336-360. http://dx.doi.org/10.1017/jfm.2011.166.

18. Mullakaev, M.S.; Abramov, O.V.; Abramov, V.O.; Gradov, O.M.; Pechkov, A.A. 2009. An ultrasonic technology for productivity restoration in low flow boreholes, Journal of Chemical and Petroleum Engineering 45: 203-210.

http://dx.doi.org/10.1007/s10556-009-9171-6.

19. Hamidi, H.; Rafati, R.; Bin Junin, R.; Manan, A.M. 2012. A role of ultrasonic frequency and power on oil mobilization in underground petroleum reservoirs, Journal of Petrol. Explor. Prod. Technol. 2: 29-36. http://dx.doi.org/10.1007/s13202-012-0018-x.
20. Blake, J.R.; Taib, B.B.; Doherty, G. 1986. Transient cavities near boundaries. Part 1. Rigid boundary, Journal of Fluid Mechanics 170: 479-497. http://dx.doi.org/10.1017/S0022112086000988.

21. Best, J.P. 1991. The dynamic of underwater explosions, Doctor of Philosophy thesis, Department of Mathematics, University of Wollongong.

22. Rayleigh, L. 1917. On the pressure developed in a liquid during collapse of a spherical void, Phil. Mag. 34: 94-98.

http://dx.doi.org/10.1080/14786440808635681.

F. Rambarzin, M. T. Shervani-Tabar, M. Taeibi-Rahni, S. A. Tabatabaie Negad

\section{ENERGY TRANSFER IN A LIQUID FILLED ELEMENTAL PASSAGE OF A POROUS MEDIUM FOR PERMEABILITY ENHANCEMENT DUE TO PULSATION OF A VAPOR BUBBLE}

S u m m a r y

In this paper, a novel method which has been proposed during the last decade for increasing of the permeability of porous media of petroleum reservoirs by transferring of energy via ultrasound waves is investigated numerically. Increasing of permeability of porous media of petroleum reservoirs results in enhancing of oil recovery. This technique is based on the idea of transferring of energy to the liquid filled porous media via the ultrasound waves and consequently producing of pulsating vapor bubbles. The generated vapor bubbles transfer the energy of ultrasound waves in the liquid filled passages of a porous medium through velocity and pressure fields in the liquid domain and in turn apply impact forces on obstacles which have been made from accumulation of clays inside passages of the porous medium around the wellbore of an oil well after a long operating time. Removing of these obstacles, results in increasing of permeability of the porous medium and enhancing of oil recovery. The boundary integral equation technique is employed for numerical simulation of the growth and collapse of the cavitation bubbles and transferring of energy in the liquid domain by the bubbles pulsations. Three different geometries have been proposed as elemental passages of a porous medium. Numerical results show that the controlled growth and collapse of vapor bubbles induced by emission of the ultrasound waves in a liquid filled porous medium could lead to a powerful technology for enhancing oil recovery and consequently for industrial and economical development of the nations and countries having petroleum and other liquid reservoirs.

Keywords: Porous medium, Cavitation vapor bubble, Boundary integral equation technique, Enhanced oil recovery (EOR), Ultrasound waves.

Received November 29, 2015 Accepted January 06, 2016 